\title{
Presentación del editor invitado Reflexiones filosóficas sobre el color
}

Luis Xavier López Farjeat

Facultad de Filosofía y Ciencias Sociales, Escuela de Filosofía

Universidad Panamericana, Ciudad de México

llopez@up.edu.mx

En De anima II aparece una de las tesis más conocidas de la teoría aristotélica de la percepción: a cada sentido le corresponde un sensible. En el De Sensu se reúnen una serie de observaciones complementarias como, por ejemplo, qué es lo que ha de decirse de cada uno de los sensibles. En términos más concretos: qué es el color, qué el sonido, qué el olor, el sabor y lo táctil. Aristóteles formula una compleja teoría del color a partir de la pregunta más elemental: ¿qué es el color y cómo es que se produce la sensación de la vista a partir del color? Al componer su teoría de la percepción del color, Aristóteles recurre a la noción de «lo transparente» (to diaphanes):

(...) aquello a lo que llamamos «transparente» no es privativo del aire o del agua ni de otro de los cuerpos así llamados, sino que es una naturaleza y una potencia (dýnamis) común que no es separable, sino que se halla en ellos y se da en los demás cuerpos, en unos más y en otros menos (De Sensu III, 439a 20-25). 
La transparencia es una propiedad de los cuerpos. Para nosotros es evidente que el aire y el agua lo son. No obstante, según Aristóteles también el resto de los cuerpos son transparentes en alguna medida. La definición del color tal como aparece en el De anima se construye a partir de lo transparente. De acuerdo con Aristóteles, lo visible es el color y de hecho es el color el que recubre a todas las cosas que son visibles «por sí». Por ello,

todo color es un agente capaz de poner en movimiento a lo transparente en acto y en esto consiste su naturaleza. He ahí -continúa Aristóteles- por qué el color no es visible si no hay luz, mientras que, a la luz, cualquier color de cualquier cosa resulta visible (De anima II, 418b1-4).

Si el color mueve a lo transparente para que sea actualizado, ello supone, como explica el propio Aristóteles, que hay cuerpos transparentes que, de algún modo, «reciben» el color:

Y llamo «transparente» a aquello que es visible si bien - por decirlo en una palabra - no es visible por sí, sino en virtud de un color ajeno a él. Tales son el aire, el agua y multitud de sólidos: no son transparentes, en efecto, ni en tanto que agua ni en tanto que aire, sino porque en ellos se da una cierta naturaleza, la misma que se da en el cuerpo eterno situado en la región más alta del firmamento (De anima II, 418b 5-10).

Lo transparente no es visible por sí, pero es algo a través de lo cual vemos los colores. Ahora bien, si lo transparente se da en multitud de sólidos, entonces, así como los cuerpos tienen un límite, lo transparente también. De acuerdo con 
Aristóteles, el color está en el límite del cuerpo, mas no es el límite del cuerpo:

Dado que el color se halla en el límite del cuerpo, estaría en el límite de lo transparente, de manera que el color sería el límite de lo transparente en un cuerpo determinado y se da en el límite en todos los cuerpos transparentes de modo semejante al del agua o cualquier otro de tal clase. Así pues, puede ocurrir que lo que produce la luz en el aire se halle en lo transparente, y puede ocurrir que no se halle, sino que el cuerpo esté privado de ello. Así pues, de la misma manera que en aquel caso se dan la luz y la oscuridad, se producen en los cuerpos lo blanco y lo negro ( $D e$ Sensu III, 439b 10).

La luz es indispensable para actualizar la dýnamis de lo transparente. La luminosidad permite que los cuerpos sean visibles o, en otros términos, que el color sea visible. Si no hay luminosidad el color no es percibido. Por ello, «la luz es el color de lo transparente por accidente - pues, cuando hay algo ígneo en lo transparente, su presencia es luz, y su ausencia, oscuridad—» (De Sensu III, 439a 20). En otras palabras, la presencia de la luz actualiza a lo transparente y posibilita la percepción del color; al contrario, si no hay actualización de lo transparente, lo que hay es oscuridad.

La descripción aristotélica es fascinante: sin color, no podríamos ver ningún cuerpo; sin embargo, el color no está en realidad en los cuerpos sino que se percibe con ocasión de la luz. Tal parece que Aristóteles no admite la materialidad del color. Sin embargo, tampoco es seguro que su teoría de la percepción aporte una respuesta satisfactoria a la pregunta por la naturaleza del color: no es claro si el órgano de la vista se ve afectado por el color, o si la captación de lo coloreado 
por mediación de la luz sugiere que es posible interpretar la teoría aristotélica de la percepción como si apuntase a la «desmaterialización» de la percepción.

En cualquier caso, no tengo la intención de resolver el problema del color en la filosofía aristotélica. Simplemente he recurrido a esta breve descripción de la problemática con la finalidad de introducir un problema discutido en la tradición filosófica: ¿qué es el color? Muchos físicos, químicos y filósofos han tratado de responder. Y posiblemente todos tengan algo de razón. Sin embargo, cada teoría, cada filosofía del color, arroja nuevas interrogantes y plantea dificultades. En este volumen hemos querido abordar el problema del color o de la percepción del color bajo la óptica de pensadores cuyas aportaciones en esta materia han sido poco exploradas: Teofrasto, René Descartes, Isaac Newton, Thomas Reid, Arthur Schopenhauer y algunos fenomenólogos del siglo xx, como Wilhelm Schapp o Adolf Reinach — por mencionar tan sólo dos de los muchos nombres que aparecen en el agudo análisis elaborado por Alejandro Vigo sobre filósofos pertenecientes a la tradición husserliana y post-husserliana.

André Laks examina a detalle una serie de fragmentos de Teofrasto sobre la doctrina de la sensación en Aristóteles. El problema al que se enfrenta Teofrasto es el siguiente: ¿qué querrá decir que la sensación es la asimilación de lo sentido por lo sensible?, ¿se da dicha asimilación en el órgano sensorial? En otras palabras: ¿será que el color «afecta» a la vista? ¿O será que lo que se recibe en la sensación es la forma? La búsqueda de una respuesta a estas interrogantes hace pensar, como anota el profesor Laks, que el papel de Teofrasto es esencial en el debate — también contemporáneo- sobre la supuesta «desmaterialización» de la percepción sensible en Aristóteles. 
«El color en El Mundo cartesiano» es un artículo de María Isabel Gamboa que analiza la explicación mecanicista de la percepción del color en El Mundo o Tratado de la luz de René Descartes. Gamboa reconoce las aportaciones cartesianas y argumenta que el comprender todo fenómeno óptico desde interacciones mecánicas permitió a Descartes alejarse de la visión aristotélica realista de los colores. El trabajo de Gamboa muestra el modo en que Descartes traslada este problema a la geometría y a las matemáticas, inaugurando con ello un modo distinto de entender la percepción del color.

En «Notas epistemológicas en torno a las investigaciones newtonianas sobre la luz y el color», Leonardo Ruiz revisa los primeros textos de Isaac Newton en donde aparece su teoría de la luz y del color. Ambas teorías evolucionaron paulatinamente. Sin embargo, su versión definitiva presentada ante la Royal Society en 1672 no escapa a una serie de consideraciones extra-fenoménicas y extra-experimentables que, según Ruiz, ponen en jaque la propia postura newtoniana.

Sin duda, una de las teorías del color más sugerentes y menos conocidas es la de Thomas Reid. En la sección IV de Una investigación de la mente humana, según los principios del sentido común, el pensador escocés argumenta que el color es una cualidad de los cuerpos y no una sensación mental. En «La aproximación sensocomunista de Thomas Reid al tema de los colores», José Hernández Prado revisa las observaciones de Reid sobre el color y sugiere que en ella ya se vislumbra lo que los teóricos contemporáneos denominan aspecto objetivo (física del color) y aspecto subjetivo (percepción visual de los colores). Pero además, el análisis de Hernández Prado deja ver cómo Reid formula, con ocasión del color, una serie de argumentos críticos contra el idealismo y el representacionismo. 
En 1810, Goethe publicó su tratado titulado Zur Farbenlehre (Teoría de los colores). Con la intención de respaldar los postulados básicos de la teoría de Goethe y con ello ganar el apadrinazgo del escritor, en 1815 Schopenhauer redactó Über das Sehn und die Farben (Sobre la visión y los colores). La teoría schopenhaueriana de los colores, tal como explica Eduardo Charpenel en «Schopenhauer y el idealismo del color», fue desatendida por mucho tiempo a pesar de que en ella hay material sumamente valioso. Schopenhauer logra articular su teoría de la visión con una descripción idealista del color, contrastante con la de Thomas Reid, por ejemplo. Creer que los colores son propiedades reales de los objetos es una postura, —según Schopenhauer- dogmática: el color es una sensación que se explica a partir de que la luz permite la actividad de la retina. Tras un análisis detallado de la concepción schopenhaueriana del color, Charpenel apunta sus atinos y desatinos.

Finalmente, en «Colores verdaderos. Una panorámica fenomenológica», Alejandro Vigo desarrolla ampliamente lo que él mismo denomina «concepciones fenomenológicas del color», elaboradas durante las primeras décadas del siglo xx. Vigo traza las coordenadas generales de las teorías del color y los problemas filosóficos que plantean autores muy cercanos a Husserl (Schapp y Reinach) y, posteriormente, hace una detallada revisión de algunos representantes de corrientes psicológicas afines a la fenomenología (Carl Stumpf, Kurt Koffka, Karl Bühler, Ewald Hering, David Katz, Adhemar Gelb, y otros). Finalmente, analiza dos de las posturas más representativas de la fenomenología post-husserliana: la de MerleauPonty y la de Hermann Schmitz. Dice Vigo que su recorrido no es exhaustivo. Discrepo: es exhaustivo y, además, ha incluido un apéndice en el que explica la teoría de Aristóteles y la de Goethe como dos posturas ya fenomenológicas. 
Agradezco a los colaboradores estas reflexiones filosóficas sobre el color. Agradezco la ayuda de Venancio Ruiz y Mario Gensollen en la lectura final de los artículos. Y agradezco también a la revista Euphyía la invitación que me ha hecho para editar este volumen.

Barrio de Mixcoac, Ciudad de México, Otoño de 2011 
